\title{
The effect of balloon dilatation eustachian tuboplasty combined with grommet insertion on the structure and function of the eustachian tube in patients with refractory otitis media with effusion
}

\author{
$\mathrm{Li} \mathrm{Li}^{1,2}$, Yanyan Mao ${ }^{1,2}, \mathrm{Na} \mathrm{Hu}^{3}$, Wenqing Yan ${ }^{1,2}$, Yanqing $\mathrm{Lu}^{1,2}$, Zhaomin Fan $^{1,2}$, Haibo Wang ${ }^{1,2}$, \\ Yuechen Han ${ }^{1,2}$
}

${ }^{1}$ Department of Otolaryngology-Head and Neck Surgery, Shandong Provincial ENT Hospital, Cheeloo College of Medicine, Shandong University, Jinan, China; ${ }^{2}$ Department of Otology Surgery, Shandong Provincial ENT Hospital, Jinan, China; ${ }^{3}$ Department of Medical Imaging Center, Shandong Provincial ENT Hospital, Jinan, China

Contributions: (I) Conception and design: L Li, Y Han; (II) Administrative support: Y Han; (III) Provision of study materials or patients: Y Mao, N Hu, W Yan; (IV) Collection and assembly of data: Y Lu, N Hu (V) Data analysis and interpretation: Z Fan, H Wang, Y Han; (VI) Manuscript writing: All authors; (VII) Final approval of manuscript: All authors.

Correspondence to: Yuechen Han. Department of Otolaryngology-Head and Neck Surgery, Shandong Provincial ENT Hospital, Cheeloo College of Medicine, Shandong University, Jinan 250022, China. Email: hanyuechen123@126.com.

\begin{abstract}
Backgroundk The treatments for refractory secretory otitis media with effusion usually choose long-term grommet insertion. This study evaluated the effect of balloon eustachian tuboplasty combined with grommet insertion on the function and the opening length of the eustachian tube in patients with refractory otitis media with effusion.
\end{abstract}

Methods: Fifty-seven patients with refractory otitis media with effusion were enrolled. A threedimensional reconstruction of an iohexol-enhanced computed tomography image was applied to evaluate the structural and length changes of the eustachian tube at both resting and Valsalva maneuver states. The grommet was removed 3 months after the operation and postoperative follow-up was carried out from 3 to 12 months. We performed pre- and post-operative observation of the following: appearance of the tympanic membrane, pure-tone audiometry threshold, eustachian tube score, seven-item Eustachian Tube Dysfunction Questionnaire scores (ETDQ-7), quantitative examination of eustachian tube function dynamic observation of tympanogram peak pressure point, and computed tomography examination of the eustachian tube.

Results: The pure-tone audiometry at 1, 3, 6, 9, and 12 months postoperatively were all significantly lower compared to the preoperative value (all $\mathrm{P}<0.05$ ). There was no significant difference between the pure-tone audiometry at 6 and 9 months postoperatively, neither was for the air-bone conduction gap at these time points. The quantitative examination peak pressure deviation was markedly increased at 6 months postoperatively compared with that before the operation (all $\mathrm{P}<0.05$ ). The peak pressure deviation of tympanometry at 6 and 9 months postoperatively were both higher than the value at 12 months after surgery $(\mathrm{P}<0.05)$. The eustachian tube score at $1,3,6,9$, and 12 months postoperatively were notably higher than that before the operation (all $\mathrm{P}<0.05$ ). A significant difference was also observed between the 6- and 12-month postoperative eustachian tube score $(\mathrm{P}<0.05)$. There was a significant difference in the ETDQ7 scores at 6 - and 12 -month postoperatively $(\mathrm{P}<0.05)$. The quantitative examination peak pressure deviation and eustachian tube score were both correlated with development length of the eustachian tube after threedimensional computed tomography reconstruction $(\mathrm{P}<0.05)$.

Conclusions: Eustachian tube balloon dilatation combined with grommet insertion is an effective treatment for refractory otitis media with effusion.

Keywords: Eustachian tube; otitis media with effusion; grommet insertion; balloon eustachian tuboplasty 
Submitted Apr 27, 2021. Accepted for publication Jun 24, 2021.

doi: 10.21037/apm-21-1280

View this article at: https://dx.doi.org/10.21037/apm-21-1280

\section{Introduction}

Refractory otitis media with effusion (OME) is characterized as continuous OME with a history of grommets insertion several times $(1,2)$. OME is a disease with adult prevalence of around $0.6 \%$, compared with incidence about $90 \%$ of children before school $(3,4)$. Most children with OME could resolve spontaneously within 3 months, except that about $5 \%$ to $10 \%$ of children have repeated OME episodes lasting $\geq 1$ year (4). OME may be caused mainly by Eustachian tube dysfunction (ETD), an ear infection (acute otitis media), some syndromes such as Down's syndrome and cleft palate which might affect the shape of the face and skull base, secondary to subclinical bacterial infection, and partly in association with gastroesophageal reflux disease (3). The treatments for refractory otitis media with effusion usually choose long-term grommet insertion $(4,5)$. However, repeated ventilation tube insertion is not successful treatment option for recurrent OME, which is difficult to cure, even might cause tympanic membrane perforation, infection, and tympanosclerosis. Unfortunately, repeated attacks of symptoms such as hearing loss, aural fullness, otalgia, tinnitus, feeling of pressure, or popping in the ears seriously affects the quality of life of patients (3-6). ETD may lead to recurrent or persistent OME. Tympanic tube insertion surgery can not directly resolve ETD and may be associated with complications [such as infection, persistent perforation, and tympanosclerosis (7)]. Thus, a new balloon dilation of the eustachian tube technique [known as balloon eustachian tuboplasty (BET)], which is a safe and effective treatment option of ETD, was first applied clinically in patients in 2010 (8). The BET technique was performed with superior postoperative outcomes compared to other medical interventions, such as the placement of tympanostomy tubes, and other conventional medical treatments, including nasal steroids, decongestants, or antihistamines (9).

BET has been a recent development for the treatment of ETD. However, Schröder et al. found that BET surgery did not achieve significant symptom improvement in OME, with an effective rate of only $66 \%$ (10). Some studies have suggested that the severely thickened mucosa of the middle ear might be one of the reasons for the unsatisfactory result of BET in some patients (11). Meanwhile, other studies have reported short-term symptom relief and a declining effective rate in the long-term effect ( $\geq 3$ years) (12-14). One previous study reported the effect of balloon dilatation eustachian tuboplasty combined with tympanostomy tubes in children patients with recurrent otitis media with effusion, which indicated that ETS was improved after the treatment. However, there is still lacking more comprehensive evaluated indicator, particularly for the adults (15). In this study, we aimed to evaluate the effect of BET combined with grommet insertion on the function and the opening structure of the eustachian tube. Assessment and quantification of Eustachian tube function involved subjective or objective methods before and after surgery. The subjective methods included an assessment of patient symptoms or the use of the seven-item Eustachian Tube Dysfunction Questionnaire scores (ETDQ-7). The objective methods included the appearance of tympanic membrane, pure-tone hearing threshold, eustachian tube score (ETS), quantitative examination of eustachian tube function (dynamic observation of tympanogram peak pressure point), and the results of computed tomography (CT) examination of the eustachian tube.

We present the following article in accordance with the STROBE reporting checklist (available at https://dx.doi. org/10.21037/apm-21-1280).

\section{Methods}

\section{Materials}

All procedures performed in this observational study involving human participants were in accordance with the Declaration of Helsinki (as revised in 2013). The study was approved by Shandong Provincial ENT hospital ethics committee (registration number of the ethics board: XYK20161201), and informed consent was taken from all the patients. We undertook this study enrolled 57 adult patients with refractory OME between January 1, 2017, and April 1, 2019. Study participants were recruited from the ENT (ear, nose and throat) clinic at Shangdong Provincial ENT Hospital affiliated to Shandong University, and included 33 male patients and 24 female patients. The 
Table 1 Eustachian tube score (ETS) (8)

\begin{tabular}{lccc}
\hline & & Score & \\
\cline { 2 - 4 } Symptom/finding & 2 & 1 & Never \\
Clicking sound when swallowing & Always & Sometimes & Never \\
Positive subjective Valsalva & Always & Sometimes & No $R$ \\
TMM 30 mbar & $R \leq 1$ & No $R$ \\
TMM 40 mbar & $R \leq 1$ & $R>1$ & No R
\end{tabular}

Eustachian tube score ranging from 0 to 10 points. TMM, tubomanometry.

mean age of study participants was 35.3 years (range, 1866 years). The follow-up time was 12 months postoperatively.

\section{Study methods}

The clinical assessment included examination of the appearance of the tympanic membrane with an otoendoscope, as well as pure-tone hearing threshold evaluation. Eustachian tube function was evaluated via the ETS, quantitative examination of eustachian tube function (dynamic observation of tympanogram peak pressure point), Valsalva's manoeuvre, Toynbee's manoeuvre, and the results of CT examination of the eustachian tube.

The following parameters were used: pure-tone audiometry (PTA): 500, 1,000, 2,000, and 4,000 Hz; air conduction and bone conduction gap (ABG): the gap of auditory thresholds between air conduction and bone conduction at 500, 1,000, 2,000, and 4,000 Hz for PTA.

\section{Quantitative examination of eustachian tube function (dynamic observation of tympanogram peak pressure point, when the tympanic membrane is intact)}

(I) The first tympanogram records resting middle-ear pressure.

(II) Valsalva's manoeuvre, and then record the second tympanogram.

(III) The subject swallows several times, and then record the third tympanogram.

When eustachian tube function is normal, the peak pressure point of the second curve in the acoustic admittance chart usually deviates more than 10-15 daPa. Peak pressure point movement of less than $10-15 \mathrm{daPa}$ or no movement is indicative of ETD.

\section{Modified inflation-deflation test (when the tympanic membrane is not intact)}

An open pressure (less than $250 \mathrm{mmH}_{2} \mathrm{O}$ ) signifies that the eustachian tube is unobstructed. If the eustachian tube opens only between $250 \mathrm{mmH}_{2} \mathrm{O}$ and $360 \mathrm{mmH}_{2} \mathrm{O}$, it can be assumed that partial mechanical obstruction is present. A high opening pressure $\left(>360 \mathrm{mmH}_{2} \mathrm{O}\right)$ may indicate total obstruction. Meanwhile, a low opening pressure $\left(<100 \mathrm{mmH}_{2} \mathrm{O}\right)$ is indicative of a semipatulous eustachian tube.

\section{ETS}

The ETS is a tool that combines both the subjective and objective elements for the assessment of eustachian tube function, including tubomanometry (TMM), clicking sound when swallowing, and positive subjective Valsalva. The ETS ranges from 0 to 10 points, with a score of 0 denoting no tubal function and the maximum score of 10 signaling the highest tubal function (8) (Table 1). An ETS $\leq 5$ suggests diagnosis of chronic ETD.

\section{The seven-item Eustachian Tube Dysfunction Questionnaire (ETDQ-7)}

ETDQ-7, a valid and reliable symptom score, was used to quantify the ETD symptoms (16) (Table 2).

Three-dimensional reconstruction of iohexol-enhanced CT image was used to evaluate the structural and length changes of the eustachian tube at resting and Valsalva maneuver states. In addition, the absence of internal carotid artery (ICA) dehiscence into the eustachian tube lumen was confirmed by a CT scan involving the temporal bone. 
Table 2 Eustachian Tube Dysfunction Questionnaire-7 (ETDQ-7) (16)

\begin{tabular}{lccc}
\hline $\begin{array}{l}\text { Over the past month, how much has each of the following } \\
\text { been a problem for you? }\end{array}$ & No problem & Moderate problem & Severe problem \\
\hline 1. Pressure in the ears? & 1,2 & $3,4,5$ & 6,7 \\
2. Pain in the ears? & 1,2 & $3,4,5$ & 6,7 \\
3. A feeling that your ears are clogged or "under water"? & 1,2 & $3,4,5$ & 6,7 \\
4. Ear symptoms when you have a cold or sinusitis? & 1,2 & $3,4,5$ & 6,7 \\
5. Crackling or popping sounds in the ears? & 1,2 & $3,4,5$ & 6,7 \\
6. Ringing in the ears? & 1,2 & $3,4,5$ & 6,7 \\
7. A feeling that your hearing is muffled? & 1,2 & $3,4,5$ & 6,7 \\
\hline
\end{tabular}

\section{Instruments and equipment}

* GE Lightspeed 64-row spiral CT scanner (GE LightSpeed Ultra multi-detector spiral CT scanner, GE Medical Systems, Milwaukee, Wisconsin, USA). A 0.625 mm-thick image was reconstructed by using the multiplanar reconstruction technique.

* Image processing software version of workstation: GE AW4.6 (GE LightSpeed Ultra multi-detector spiral CT scanner, GE Medical Systems, Milwaukee, Wisconsin, USA).

* Grayson-Stadler Audio star Pro dual-channel diagnostic audiometer (Minnesota, USA).

* GSI Tympstar otoadmittance (Minnesota, USA).

\section{Tuboplasty method}

The BET procedures were performed as previously described by Ockermann et al. (8). A Bielefeld balloon catheter (Spiggle and Theis, Overath, Germany) was used to dilate the eustachian tube. The balloon was inflated with normal saline to 10 bars (1,000 kpa) for 2-min once inside the cartilaginous part of the eustachian tube.

\section{Follow-up}

Postoperative follow-up was carried out from 1 to 12 months. Re-visits were conducted every 3 months until the function of eustachian tube was no longer changed, including at 4 months postoperation. Audiometry and tympanometry were performed, and the ETS, ETDQ-7 scores, quantitative examination of eustachian tube function (dynamic observation of tympanogram peak pressure point), and results of three-dimensional reconstruction of iohexolenhanced CT scan of the eustachian tube were obtained.

\section{Statistical analyses}

Data were analyzed using the SPSS24.0 Statistical Analysis Software (SPSS Inc., Chicago, IL, United States). The pure-tone hearing threshold, air-bone conduction gap, and open length of the eustachian tube were compared before and after surgery using the paired $t$-test. Spearman correlation analysis was used to assess the changes of the open length of the eustachian tube after three-dimensional reconstruction of the iohexol-enhanced CT scan, as well as quantitative examination of eustachian tube function. $\mathrm{P}$ value less than 0.05 was considered statistically significant.

\section{Results}

Fifty-seven patients underwent BET and grommet insertion, including 33 males and 24 females. All 57 cases were unilateral, with a mean age of 35.3 years.

The pure-tone auditory threshold at 1 day postoperatively was significantly lower than those before the operation $(\mathrm{P}<0.05)$, and then remained stable and decreased slightly at 9 months postoperatively. The air-bone gap exhibited the same result. The ventilation tube was removed at 3 months after operation (the eustachian tube function was stable). There was no significant difference between the pure-tone auditory thresholds at 6- and 9-month postoperatively, and the same result was observed for the air-bone conduction gap (Figure 1).

The quantitative examination peak pressure deviation in 


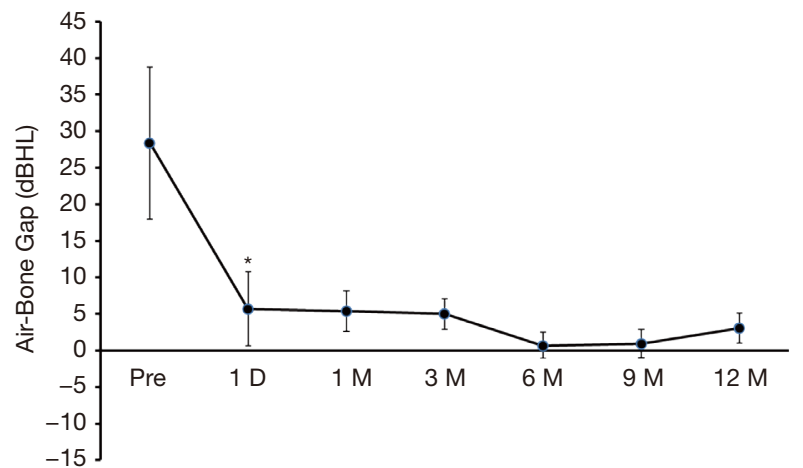

Figure 1 Air-bone gap. *, $\mathrm{P}<0.05$.

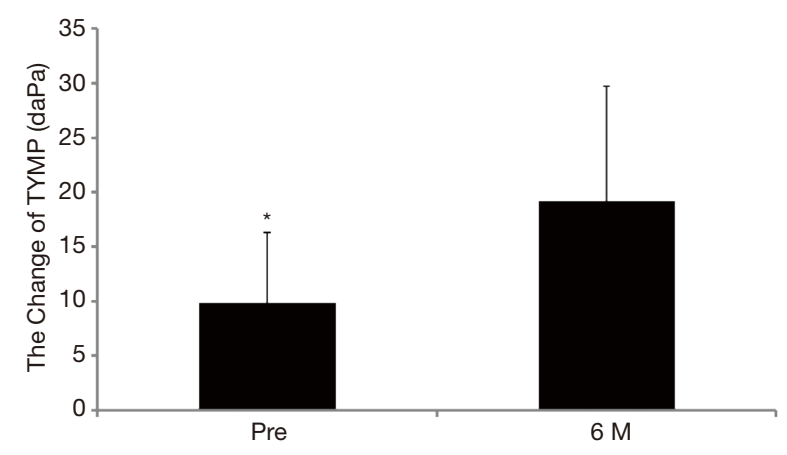

Figure 2 The quantitative examination peak pressure deviation in tympanogram. *, $\mathrm{P}<0.05$.

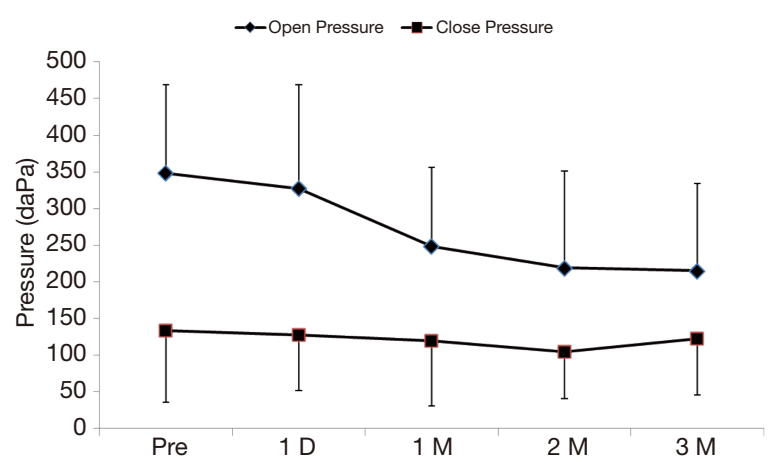

Figure 3 The open and close pressures of eustachian tube.

the tympanogram of eustachian tube function was less than $10 \mathrm{daPa}$ preoperatively, and was significantly increased at 6 months post-surgery $(\mathrm{P}<0.05)$, with the deviation being greater than $15 \mathrm{daPa}$ (Figure 2). There was a significant difference in the quantitative examination peak pressure deviation and ETS between 4- and 6-month postoperatively.

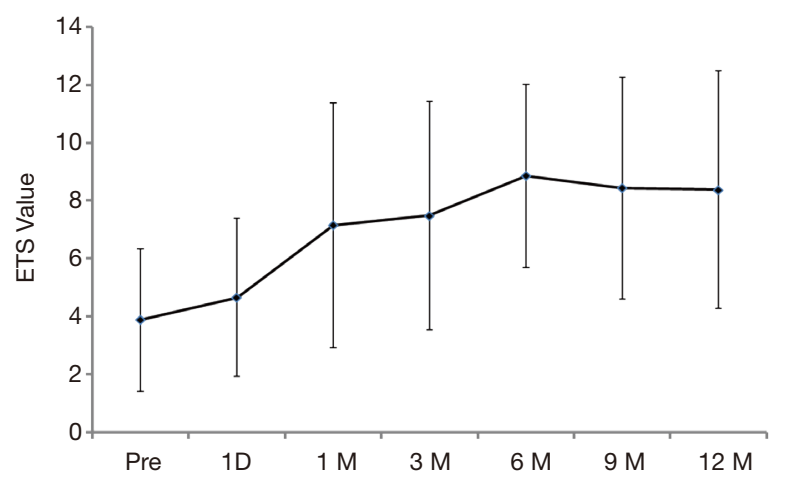

Figure 4 Changes in the ETS. ETS, eustachian tube score.

The peak pressure deviation of tympanometry at 6 and 9 months postoperatively were both higher than the value at 12 months after surgery $(\mathrm{P}<0.05)$.

Regarding the subjects who received BET combined with grommet insertion, the open pressure of the eustachian tube was still higher than $360 \mathrm{mmH}_{2} \mathrm{O}$ at 1 day postsurgery. This gradually decreased and reached a stable level at 3 months postoperatively, which suggest that the tube can be removed at this time (Figure 3). The tympanic ventilation tube was removed at 3 months postoperatively, however OME occurred in five patients at 9 months and in seven patients at 12 months after the operation. The close pressure did not change obviously.

The ETS was improved at 1-month post-surgery, and was stable from 3 months postoperatively. The ETS at 1, 3, 6,9 , and 12 months postoperatively was significantly higher than that before surgery (all $\mathrm{P}<0.05$ ). There was a marked difference between the ETS at 6- and 12-month after the operation $(\mathrm{P}<0.05)$ (Figure 4$)$.

There was a significant difference in the ETDQ-7 scores at 6- and 12-month postoperatively $(\mathrm{P}<0.05)$ (Figure 5). The quantitative examination peak pressure deviation of eustachian tube function and ETS were both correlated with the change in the development length of the eustachian tube according to the three-dimensional CT reconstruction $(\mathrm{P}<0.05)$.

At the 6-month follow-up, the overall number of BET combined with grommet insertion subjects with normalized tympanograms and those with normalized ETDQ-7 scores remained unchanged relative to those at 4 months.

At the 12-month follow up, the air-bone gap increased in seven patients $(12.3 \%)$, the quantitative examination peak pressure deviation decreased in nine patients (15.8\%), the ETS decreased in 10 patients (17.5\%), and the ETDQ-7 


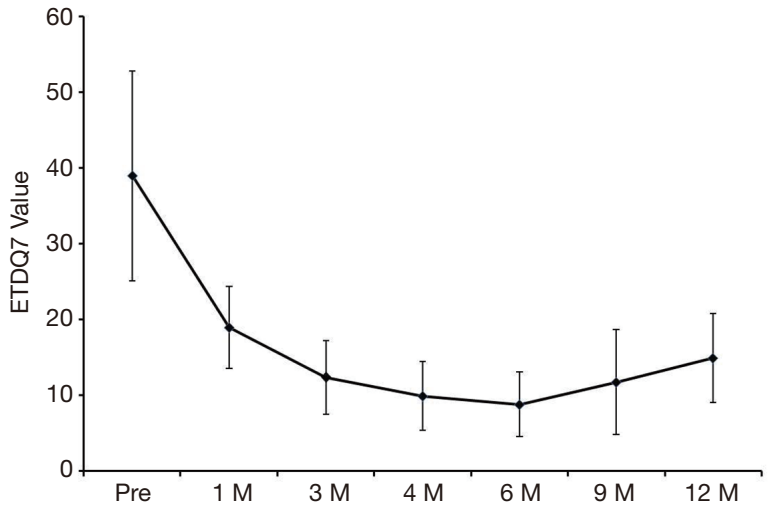

Figure 5 Changes in the ETDQ-7 scores. ETDQ-7, seven-item Eustachian Tube Dysfunction Questionnaire scores.

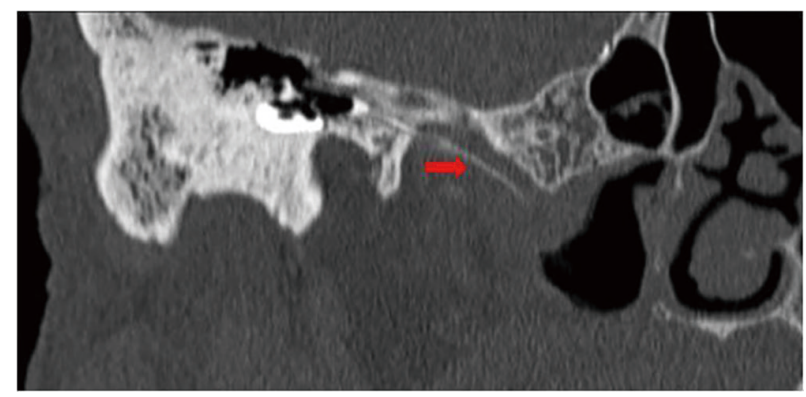

Figure 6 CT (oblique coronal position) iohexol imaging, 3 months after eustachian tube balloon dilatation. Red arrow represents the eustachian tube enhanced by iohexol contrast medium.

Table 3 Comparison of the developing length of contrast medium in the eustachian tube before and after surgery (mean $\pm \mathrm{SD}$ )

\begin{tabular}{lc}
\hline Time & $\begin{array}{c}\text { lohexol developing length of } \\
\text { eustachian tube }\end{array}$ \\
\hline Before surgery & $11.89 \pm 3.15$ \\
3-month postoperatively & $30.35 \pm 16.12$ \\
\hline
\end{tabular}

scores became abnormal in 12 patients (21.1\%).

The developing length of contrast medium 3 months postoperatively (Figure 6) significantly increased compared with that before surgery $(\mathrm{P}<0.01)$ (Table 3, Figure 7). There was a linear correlation between the developing length of the contrast medium and the ETS, $\mathrm{r}=0.81, \mathrm{P}<0.05$.

\section{Discussion}

The eustachian tube is a complex osseocartilaginous

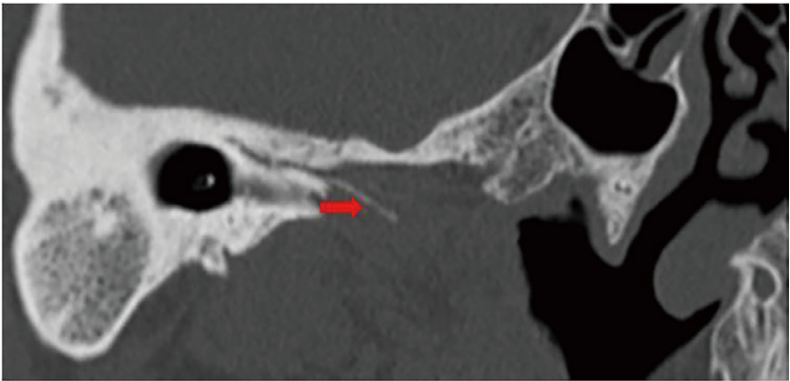

Figure 7 CT (oblique coronal position) iohexol imaging before eustachian tube balloon dilation. Red arrow represents the eustachian tube enhanced by iohexol contrast medium.

connection between the protympanum and the nasopharynx. It is an organ consisting of a lumen with its mucosa, cartilage, surrounding soft tissue, paratubal muscles, and bony support. The eustachian tube is divided into three portions: cartilaginous, junctional, and osseous. The cartilaginous portion opens into the nasopharynx (17).

Failure of the eustachian tube to function correctly is a common cause of morbidity. ETD may lead to recurrent or persistent OME, hearing loss, temporary lag in speech development in children, retraction of the tympanic membrane, or even cholesteatoma. Treatments such as the placement of tympanostomy tubes create an alternative route for ventilation of the middle ear space, but do not directly address the problem of the eustachian tube (7). BET aims to improve the ventilatory and pressure-equalising function of the eustachian tube in order to prevent, reverse, or stop the progression of ETD conditions by widening the medial cartilaginous part of the eustachian tube. A histological study of BET suggested that balloon-mediated compression of irreversibly injured hypertrophic mucosa, submucosal inflammatory lymphoid infiltrate, and follicular hyperplasia may allow for a replacement layer of thinner and healthier layers (9). Kivekäs et al. also found that diffuse crush injuries and shearing of the mucosa and submucosa were restored to largely healthy ciliated pseudo-columnar epithelium at 5-12 weeks postoperatively following BET (18). It is likely that BET removes irreversibly injured or inflamed mucosal and submucosal tissue, promoting recovery with normal cell lining (19). Therefore, with the return of the normal ciliated epithelium and reduced inflammatory infiltrate, it is possible that BET could cause some permanent changes to the cartilaginous eustachian tube that could provide lasting benefit in widening the lumen and facilitate the 
dilatory action of the tensor veli palatini (TVP) muscle (20). With the maintenance of a correct middle ear pressure, information provided by chemoreceptors and baroreceptors of the tympanic plexus is transmitted to the nuclei of the solitary tract, and then a neural circuit promotes the activation of eustachian tube muscles. Perturbations of the middle ear pressure may also stimulate saliva production, with consequent swallowing, opening of the eustachian tube, and endotympanic pressure rebalancing (21).

In our study, patients treated with BET and grommet insertion had a satisfying outcome in terms of their subjective symptoms and objective evaluations, and also achieved good results in eustachian tube function and structure, which is consistent with the findings of Yin et al. (22). The quantitative examination peak pressure deviation of eustachian tube function significantly increased postoperatively $(\mathrm{P}<0.05)$, as did the ETS. After the operation, the pure-tone auditory threshold and air-bone gap were significantly lower than those before surgery $(\mathrm{P}<0.05)$. There was no significant difference in the pure-tone auditory threshold between 3- and 6-month postoperatively. Meanwhile, there was a significant difference in the quantitative examination peak pressure deviation and ETS between 4- and 6-month postoperatively. The reason for this may be that although the function of the eustachian tube gradually recovered, there was no inflammatory change or effusion in the tympanum. However, there was an inflammatory reaction and scar formation after physical extrusion of the eustachian tube mucosa, submucosa, and peri-eustachian tube at 3 months after surgery. The function of eustachian tube was not restored to normal until 6 months postoperatively.

Given that visualizing the eustachian tube lumen could perhaps provide information on its patency and the level of obstruction, and radiological assessment of the eustachian tube could provide a useful non-invasive solution to assessing eustachian tube function (23), we applied iodinebased contrast mediums to visualize the eustachian tube. The length of the contrast agent of eustachian tube was significantly increased both before and after the operation. This is related to the enlargement of the cartilaginous segment of eustachian tube, the increase of the patency of eustachian tube, and the improvement of the active and passive opening function of the eustachian tube. At the same time, it may also be that in the inflammatory state of the middle ear, the secretion of the glands in the eustachian tube increases. Following BET, the autonomic nerves in the mucosa and surrounding tissues of the eustachian tube are damaged, and thus, that the effect of promoting secretion and vasodilation is weakened.

In our study, at the 12-month follow up, the air-bone gap increased in seven patients $(12.3 \%)$, the quantitative examination peak pressure deviation decreased in nine cases (15.8\%). the ETS decreased in 10 patients (17.5\%), and the ETDQ-7 scores became abnormal in 12 cases (21.1\%). These results indicate that the function of eustachian tube decreased, and that not all patients ultimately benefit from BET (24-26). Of the 57 patients/ears, OME recurred after BET in seven cases. Among these seven cases, one case had allergic rhinitis and asthma, which may explain the fact that the operation did not treat the allergic inflammatory reaction in the middle ear and eustachian tube. Therefore, treating the allergic diseases that affect the function of eustachian tube and removing the obstruction is key.

In the remaining six cases, although there were no allergic diseases of the nose and respiratory tract, the recurrence of OME after BET may be related to the following: the insufficient traction force after the formation of the soft tissue scar around the eustachian tube caused by the single BET operation, the insufficient opening of lumen, the weakening of passive opening ability, and the insufficient reduction of impedance to the tensor veli palatine muscle. Six cases got better improvement after the combined treatment, unfortunately, they recurrented finally. Even after recurrence, the symptom was better than that before the combined treatment. Also, despite the fact that OME recurred after the operation, both the Valsava and Toynbee maneuvers were easier than before, and eustachian tube function was partially improved. In these patients, perhaps appropriate hyperbaric dilation, delayed dilation, and repeated dilation are effective methods to solve these problems.

After balloon dilatation, the eustachian tube was enlarged and the development length of the eustachian tube was increased. Balloon dilation can effectively improve the active and passive opening function of the eustachian tube. Eustachian tube balloon dilatation combined with grommet insertion is an effective treatment for refractory otitis media with effusion.

\section{Acknowledgments}

Funding: This study was supported by the National Nature Science Foundation of China (81970873). 


\section{Footnote}

Reporting Checklist: The authors have completed the STROBE reporting checklist. Available at https://dx.doi. org/10.21037/apm-21-1280

Data Sharing Statement: Available at https://dx.doi. org/10.21037/apm-21-1280

Conflicts of Interest: All authors have completed the ICMJE uniform disclosure form (available at https://dx.doi. org/10.21037/apm-21-1280). The authors have no conflicts of interest to declare.

Ethical Statement: The authors are accountable for all aspects of the work in ensuring that questions related to the accuracy or integrity of any part of the work are appropriately investigated and resolved. All procedures performed in this observational study involving human participants were in accordance with the Declaration of Helsinki (as revised in 2013). The study was approved by Shandong Provincial ENT Hospital ethics committee (No.: XYK20161201), and informed consent was taken from all the patients.

Open Access Statement: This is an Open Access article distributed in accordance with the Creative Commons Attribution-NonCommercial-NoDerivs 4.0 International License (CC BY-NC-ND 4.0), which permits the noncommercial replication and distribution of the article with the strict proviso that no changes or edits are made and the original work is properly cited (including links to both the formal publication through the relevant DOI and the license). See: https://creativecommons.org/licenses/by-nc-nd/4.0/.

\section{References}

1. Kutluhan A, Tarlak B, Cetin H, et al. Mastoid antral ventilation tube; new treatment modality for recurrent otitis media with effusion and its long term results. Int J Clin Exp Med 2015;8:5774-80.

2. Fujita A, Honjo I, Kurata K, et al. Refractory otitis media with effusion from viewpoints of eustachian tube dysfunction and nasal sinusitis. Am J Otolaryngol 1993;14:187-90.

3. Atkinson H, Wallis S, Coatesworth AP. Otitis media with effusion. Postgrad Med 2015;127:381-5.

4. Rosenfeld RM, Shin JJ, Schwartz SR, et al. Clinical
Practice Guideline: Otitis Media with Effusion (Update). Otolaryngol Head Neck Surg 2016;154:S1-S41.

5. Dempster JH, Swan IR. The management of otitis media with effusion in adults. Clin Otolaryngol Allied Sci 1988;13:197-9.

6. Perera R, Glasziou PP, Heneghan CJ, et al. Autoinflation for hearing loss associated with otitis media with effusion. Cochrane Database Syst Rev 2013;(5):CD006285.

7. Hwang SY, Kok S, Walton J. Balloon dilation for Eustachian tube dysfunction: systematic review. J Laryngol Otol 2016;130:S2-S6.

8. Ockermann T, Reineke U, Upile T, et al. Balloon dilatation Eustachian tuboplasty: a clinical study. Laryngoscope 2010;120:1411-6.

9. Poe D, Anand V, Dean M, et al. Balloon dilation of the eustachian tube for dilatory dysfunction: A randomized controlled trial. Laryngoscope 2018;128:1200-6.

10. Schröder S, Reineke U, Lehmann M, et al. Chronic obstructive eustachian tube dysfunction in adults: longterm results of balloon eustachian tuboplasty. $\mathrm{HNO}$ 2013;61:142-51.

11. Christov F, Gluth MB. Histopathology of the mucosa of eustachian tube orifice at the middle ear in chronic otitis media with effusion: possible insight into tuboplasty failure. Ann Otol Rhinol Laryngol 2018;127:817-22.

12. Schröder S, Lehmann M, Ebmeyer J, et al. Balloon Eustachian tuboplasty: a retrospective cohort study. Clin Otolaryngol 2015;40:629-38.

13. Luukkainen V, Vnencak M, Aarnisalo AA, et al. Patient satisfaction in the long-term effects of Eustachiantube balloon dilation is encouraging. Acta Otolaryngol 2018;138:122-7.

14. Poe DS, Silvola J, Pyykko I. Balloon dilation of the cartilaginous Eustachian tube. Otolaryngol Head Neck Surg 2011;144:563-9.

15. Liu W, Chen M, Hao J, et al. The short-term and longterm efficacy of balloon dilatation Eustachian tuboplasty and tympanostomy tubes in children patients with recurrent otitis media with effusion. Lin Chung Er Bi Yan Hou Tou Jing Wai Ke Za Zhi 2020;34:210-4.

16. McCoul ED, Anand VK, Christos PJ. Validating the clinical assessment of eustachian tube dysfunction: the eustachian tube dysfunction questionnaire (ETDQ-7). Laryngoscope 2012;122:1137-41.

17. Bluestone CD. Eustachian tube: structure, function, role in otitis media. Hamilton, BC Decker Inc., 2005.

18. Kivekäs I, Chao WC, Faquin W, et al. Histopathology of balloon-dilation Eustachian tuboplasty. Laryngoscope 
2015;125:436-41.

19. Smith ME, Weir AE, Prior DCC, et al. The mechanism of balloon Eustachian tuboplasty: a biomechanical study. Med Biol Eng Comput 2020;58:689-99.

20. Poe DS, Hanna BM. Balloon dilation of the cartilaginous portion of the eustachian tube: initial safety and feasibility analysis in a cadaver model. Am J Otolaryngol 2011;32:115-23.

21. Rinaldi V, Cappadona M, Gaffuri M, et al. Chorda tympani nerve, may it have a role in stabilizing middle ear pressure? Med Hypotheses 2013;80:726-7.

22. Yin G, Tan J, Li P. Balloon dilation of Eustachian tube combined with tympanostomy tube insertion and middle ear pressure equalization therapy for recurrent secretory otitis media. J Otol 2019;14:101-5.

Cite this article as: $\mathrm{Li} \mathrm{L,} \mathrm{Mao} \mathrm{Y,} \mathrm{Hu} \mathrm{N,} \mathrm{Yan} \mathrm{W,} \mathrm{Lu} \mathrm{Y,} \mathrm{Fan} \mathrm{Z,}$ Wang H, Han Y. The effect of balloon dilatation eustachian tuboplasty combined with grommet insertion on the structure and function of the eustachian tube in patients with refractory otitis media with effusion. Ann Palliat Med 2021;10(7):7662-7670. doi: 10.21037/apm-21-1280
23. Falkenberg-Jensen B, Jablonski GE, Silvola JT, et al. CT imaging of the Eustachian tube using focal contrast medium administration: a feasibility study in humans. Acta Radiol Open 2020;9:2058460119900435.

24. Llewellyn A, Norman G, Harden M, et al. Interventions for adult Eustachian tube dysfunction: a systematic review. Health Technol Assess 2014;18:1-180; v-vi.

25. Huisman JML, Verdam FJ, Stegeman I, et al. Treatment of Eustachian tube dysfunction with balloon dilation: A systematic review. Laryngoscope 2018;128:237-47.

26. Smith ME, Scoffings DJ, Tysome JR. Imaging of the Eustachian tube and its function: a systematic review. Neuroradiology 2016;58:543-56.

(English Language Editor: A. Kassem) 